\title{
LUCIEN BRUNEL,
}

Secrétaire du Service des Missions

du Comité international.

\section{Mission en Pologne.}

Chargé de me rendre à Varsovie pour étudier sur place, avec notre délégué $M$. Gloor, certaines questions intéressant tout particulièrement le service des missions du Comité international de la Croix-Rouge, je quittai Genève le 25 octobre. Obligé de passer deux jours à Vienne, j'ai pu me rendre compte une fois de plus de la grande activité qui règne à notre bureau du Parkring et de la tâche difficile qui incombe à notre mission en Autriche, dirigée actuellement par le $\mathrm{Dr}$ Ferrière, vice-président du Comité international de la Croix-Rouge.

Arrivé à Varsovie le samedi 30 octobre, je trouvai les bureaux fermés jusqu'au mardi soir, à l'occasion des vacances de la Toussaint; M. Gloor me proposa, le dimanche matin, de partir pour Otwock, ravissante localité située à l'est de Varsovie au milieu des sapins et des pins, et renommée pour son air pur et vivifiant. C'est à Otwock que M. Gloor, avec l'aide dévouée du $\mathrm{D}^{\mathrm{r}}$ et de

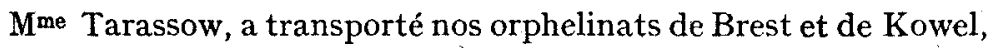
lors de l'avance des bolchéviks, au mois de juillet dernier. Nous passâmes une journée heureuse au milieu de ces 92 orphelins si bien soignés, si propres, si heureux de revivre. Cela nous procura également l'opportunitéde causer longuement avec le $\mathrm{Dr}$ Tarassow de la lutte contre le typhus, la tuberculose, le rachitisme et d'autres fléaux non moins terribles, qui ravagent actuellement tout l'Est de l'Europe.

Le général Haller nous avait accordé une audience pour le mercredi. Etant arrivés un peu avant l'heure qui nous avait été fixée, nous eûmes le privilège de nous entretenir avec la comtesse de Bisping, qui nous fit visiter les locaux de la Croix-Rouge polonaise et nous présenta aux chefs des différents départements de cette importante organisation. 


\section{Lucien Brunel.}

L'accueil que nous fit le président de la Croix-Rouge polonaise fut des plus flatteurs. Il nous dit combien la Croix-Rouge polonaise était heureuse de pouvoir travailler en étroite collaboration avec le délégué du Comité international à Varsovie, et combien personnellement il se réjouissait de constater les bonnes relations actives et suivies qui existent entre le Comité international et l'institution à laquelle il voue et son temps et ses forces. Le général Haller m'a prié de transmettre ses vœux au Comité international et de présenter ses hommages à son honorable président, M. Ador.

Le général Haller parla avec chaleur de la triste situation des prisonniers polonais en Russie, du dénûment dans lequel ils se trouvent, de leur triste sort qui ne doit pas être comparé à celui, si digne de compassion qu'il soit, des autres prisonniers internés pendant cette terrible et longue guerre. Il déplora vivement le fait que personne en Russie ne fût à même de s'occuper de ces infortunés, et il s'adressa au président du Comité international pour qu'il intercède en leur faveur et qu'il obtienne des pays moins éprouvés des envois de vêtements et de linge; ces envois pourraient être dirigés sur un de nos camps de passage et être ensuite expédiés aux prisonniers, sous contrôle.

Cette conversation nous amena tout naturellement à parler de la Croix-Rouge polonaise et de son travail. Le général Haller, qui saisit cette occasion pour nous présenter quelques-uns de ses. collaborateurs, entre autres le secrétaire général, M. Yankowski, me dit que les membres de la Croix-Rouge polonaise étaient actuellement au nombre d'un million. Beaucoup de nouvelles filiales s'organisent et dépendent du Comité central à Varsovie. La Croix-Rouge polonaise n'a plus de fonds; sa situation, vu surtout le renchérissement énorme de la vie, devient critique. Ayant installé des hôpitaux, des dispensaires, des sanatoriums, 3 maisons de santé pour les convalescents, formé 5 grands trains. sanitaires, organisé des bains pour la désinfection, elle se voit dans l'obligation de conserver un nombreux personnel.

Les nouveaux statuts de la Croix-Rouge seront soumis sous. peu à la Diète et seront envoyés au Comité international de la. 


\section{Mission \\ en Pologne.}

Croix-Rouge et aux différentes Croix-Rouges aussitôt qu'ils seront imprimés.

Nous nous entretînmes ensuite longtemps des Polonais internés en Prusse orientale, auxquels la Croix-Rouge polonaise espère pouvoir envoyer des vêtements, de l'échange des prisonniers de guerre polonais et russes, des prisonniers de guerre à Menden, des questions de finances concernant le rapatriement des prisonniers de guerre polonais par la Baltique.

Le général Haller me chargea de remercier le Comité international de la Croix-Rouge de l'appel lancé par lui en faveur de la Pologne, lors de l'avance des troupes russes sur Varsovie, appel qui a eu des résultats heureux et provoqua des envois de la part de plusieurs Croix-Rouges et sociétés philanthropiques.

Nous prîmes congé du président de la Croix-Rouge polonaise non sans lui avoir parlé auparavant de la situation des prisonniers bolchévistes en Pologne, et l'avoir prié de faciliter notre tâche en nous aidant à obtenir tous les renseignements nécessaires pour mener à bien la mission qui nous était confiée.

Les deux principaux camps de prisonniers russes en Pologne se trouvent à Strzalkowo (près de Posen) et à Dembié. Les autres, moins importants, à Pikulincé (près de Przemyschl), Demblyn, Brest-Litovsk, etc.

Le nombre des prisonniers, qui était de 130,000 au début, n'était plus, lors de mon séjour à Varsovie, en octobre, que de 60,000 environ. Le camp le plus important comprenait des prisonniers appartenant aux régions qui font partie de la Pologne actuelle, des Russes de l'ancienne armée blanche, des hommes enrôlés par le Gouvernement des Soviets, des communistes, des juifs, des Ukrainiens, des invalides et des femmes. Une centaine de ces femmes ont été envoyées à Dembié. Il en reste 53 à Strzalkowo, femmes de soldats et de fonctionnaires. Elles y sont logées dans d'assez bonnes conditions, couchent dans des lits, mais n'ont presque rien à se mettre sur le corps. 


\section{Lucien Brunel.}

La nourriture, qui est celle des soldats polonais, serait suffisante si la quantité fournie par le Gouvernement polonais était distribuée régulièrement. Le sucre, la graisse et la farine font défaut.

Ces prisonniers travaillaient aux champs, tant que la saison le permettait. Actuellement, ils sont tous rentrés dans les camps. Ils souffrent du froid, n'ayant pas de vêtements chauds, et dorment sur des planches ou sur le sol. Les paillasses et les couvertures sont en quantité tout à fait insuffisante; le savon manque complètement.

Le nombre des malades s'accroît tous les jours, et l'hôpital qui ne contient que $x, 300$ places ne suffit pas à recevoir tous les malades atteints de la dysenterie et du typhus. Un grand nombre d'entre eux sont poitrinaires et les cas de décès augmentent avec la mauvaise saison. Beaucoup de ces malheureux ne passeront pas l'hiver ou resteront invalides.

L'Union chrétienne américaine et l'American Joint Distribution Commitee s'occupent d'eux avec un zèle digne des plus grands éloges.

La question du rapatriement de ces prisonniers se traite actuellement à Riga, où le Gouvernement polonais a envoyé un délégué spécial, M. Zalecki.

Le délégué du Comité international s'occupera de faire parvenir la correspondance de ces prisonniers à leurs familles, s'il peut obtenir la réciprocité pour les prisonniers polonais internés en Russie.

Des familles entières venant de Russie et fuyant devant les troupes bolchévistes se sont réfugiées en Pologne. Le Gouvernement polonais ne peut pas les autoriser à se rendre à Varsovie, vu l'extrême pénurie de logements ; aussi ces malheureux errentils, lamentables épaves, en Wolhynie, dans les Krès, sans vêtements, ne sachant où trouver un abri et privés souvent de nourriture.

Mme Lubimoff, déléguée de la Croix-Rouge russe en Pologne, 


\section{Mission en Pologne.}

et M. Ossovietski, commissaire du Gouvernement polonais, sont venus nous entretenir de la situation tragique dans laquelle se trouvent ces familles composées surtout de vieillards, de femmes et d'enfants, et nous ont demandé de prier le Comité internatio nal de la Croix-Rouge d'intervenir en leur faveur auprès des institutions qui pourraient s'intéresser à leur sort.

Le général Niessel, que j'étais allé remercier au nom du Comité international de toute la sympathie que la mission française en Pologne avait toujours témoignée à nos délégués, me parla également en termes émus du sort de ces pauvres errants, sort d'autant plus digne de pitié que la Croix-Rouge russe, dont le dévouement est si précieux, se voit dans l'obligation de cesser son inlassable activité, faute de fonds.

Le comte Tyskiewicz, que nous eûmes le plaisir de recevoir dans les bureaux de notre mission, nous parla longtemps aussi de ces malheureux, et attira surtout notre attention sur le grand nombre d'enfants actuellement en Pologne, victimes inconscientes de cette guerre, qui, malgré le zèle infatigable des nombreuses institutions qui s'occupent d'eux, restent encore pour la plupart sans secours suffisants.

J'eus à ce propos l'honneur d'être reçu par Mgr Kakowski, primat de Pologne, que la question de secours aux enfants intéresse tout particulièrement. Mgr Kakowski a insisté sur la misère des enfants dans les grandes villes et a promis de donner à M. Gloor des indications qui permettent de distribuer les dons aux enfants de la façon la plus judicieuse.

Pour arriver à secourir ces milliers d'enfants d'une façon rationnelle, il est nécessaire de connaître exactement la situation géographique de la Pologne actuelle qui doit, à ce point de vue, être divisée en deux : d'un côté la Pologne proprement dite (formée de la Posnanie, de la Poméranie, de l'ancienne Pologne russe et des deux Galicies), et de l'autre les pays occupés actuellement par la Pologne, les Krès. C'est cette partie du pays qui a le plus souffert. Elle a été six ans durant le centre de tous les combats, a assisté aux avances et aux retraites de plusieurs 


\section{Lucien Brunel.}

armées belligérantes et a été le témoin de nombreuses guerres civiles.

Les parents ont disparu pour la plupart, soit qu'ils aient été faits prisonniers et emmenés en Allemagne ou en Russie, soit qu'ils aient été enlevés par le typhus, la dysenterie ou le choléra, maladies qui règnent encore dans ces régions. Les enfants sont pour la plupart des orphelins, et sont disséminés dans tout le pays.

De ces 2 millions d'enfants, les orthodoxes, qui sont les plus nombreux, ne sont presque pas secourus, tandis que les enfants catholiques bénéficient des dons envoyés par le Vatican, et les enfants israélites des secours fournis par les actions juives-américaines.

De nombreuses institutions s'occupent d'eux. La "Rada Glowna Opiekunska ", la plus importante, soigne à elle seule 300,000 enfants.

Le Comité de secours du gouvernement (P. R. P. D). fournit de son côté des rations, avec l'aide de l'action Hoover. Il envoie actuellement à Vilna des rations pour 10,000 enfants, et 2,000 rations pour les orphelinats.

Les dons envoyés par l'Union internationale de secours aux enfants à notre délégué sont des plus importants. Je suis allé visiter l'entrepôt qui a été mis généreusement à la disposition de notre mission par la Croix-Rouge polonaise, vaste local dans lequel sont rangés, à côté des dons de l'Union internationale de secours aux enfants, ceux reçus de la Croix-Rouge italienne, à la suite de l'appel du Comité international de la Croix-Rouge, ainsi que les 3 wagons envoyés par le Comité suisse de secours aux enfants. Les 3 wagons offerts par la Croix-Rouge française n'étaient pas encore arrivés à Varsovie, mais le général Niessel espérait les recevoir d'un jour à l'autre.

Les 3,000 fr. qui ont été remis au Comité international par la Croix-Rouge espagnole seront employés, sur la demande du général Haller, à l'achat de savon, d'iode et de différents antiseptiques.

Avant de quitter Varsovie, j'eus encore l'occasion de visiter 


\section{Mission \\ en Pologne.}

un des nombreux asiles dirigés par Mme Lubimoff, où 300 enfants reçoivent quotidiennement de la soupe et du pain.

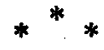

Obligé d'être rentré à Genève à date fixe, je n'eus malheureusement pas le temps de rendre visite aux représentants des Croix-Rouges et autres institutions tant locales qu'étrangères qui ont toujours accordé à notre mission de Varsovie un précieux appui. Je tiens, en terminant ce court rapport, à les en remercier au nom du Comité international de la Croix-Rouge, et certain d'être l'interprète de tous ceux qui apprécient leur beau travail, à leur adresser l'expression de toute notre reconnaissance pour l'aide apportée par eux à l'œuvre de la Croix-Rouge.

Novembre 1920. 